\title{
TRK Receptor Family Fusion Positive
}

National Cancer Institute

\section{Source}

National Cancer Institute. TRK Receptor Family Fusion Positive. NCI Thesaurus. Code C138156.

Indicates that the expression of a fusion involving a TRK receptor family member has been detected in a sample. 